\title{
Os cirurgiões e a pandemia do COVID-19.
}

\section{The surgeons and the COVID-19 pandemic.}

\author{
M. Isabel T. D. Correia ${ }^{10}$; Rodrigo Felippe Ramos²; Luiz Carlos Von Bahten ${ }^{3}$
}

R E S U M O

\begin{abstract}
A atual pandemia do novo coronavírus, Covid-19, tem sido o assunto mais discutido no momento, tanto na mídia como em periódicos científicos. O medo, a incerteza e o desconhecimento sobre o comportamento da doença são os fatores preponderantes que podem justificar essa realidade. Sabe-se de antemão que a enfermidade tem grande disseminação, é mais grave entre idosos e está associada a grande impacto socioeconômico mundial. Além dos desafios em lidar com o desconhecido, há outros relacionados com a sobrecarga de informação e nesse quesito, a grande quantidade de estudos científicos, que englobam pesquisas in vitro, relatos de casos, estudos observacionais e randomizados, além de revisões sistemáticas complementam o panorama de dúvidas. Essa situação é piorada porque o conhecimento sobre o método científico não é dominado pela maioria dos profissionais de saúde, algo que há muito já vem sendo divulgado por vários autores. De sorte que em função desta realidade, torna-se fundamental que sociedades de especialidades apoiadas por dados da Organização Mundial da Saúde e do Ministério da Saúde assumam a liderança da divulgação da informação correta e confiável. O Colégio Brasileiro de Cirurgiões sugere neste documento medidas que podem auxiliar cirurgiões e outros profissionais de saúde, assim como pacientes, em caso de necessidade cirúrgica, a lidar com a atual pandemia.
\end{abstract}

Descritores: Pandemias. Cirurgia Geral. Cuidados Pré-Operatórios. Cuidados Intra-Operatórios. Cuidados PósOperatórios. Organizações de Planejamento e Atendimento a Desastre.

A pandemia do novo coronavírus, Covid-19, é o assunto mais discutido nos dias de hoje, seja no imaginário popular, nos veículos de mídia ou nas páginas da literatura científica. Esta realidade é decorrente do medo, da incerteza e, principalmente, do desconhecimento sobre o comportamento da doença. De antemão, pode-se afirmar que tem capacidade de disseminação geométrica, grande impacto socioeconomômico mundial e taxas de letalidade, que aparentemente baixas no contexto global, parecem ser muito altas entre idosos, principalmente, na Itália.

A doença tem influenciado o cotidiano de todos de forma contundente. Desde a obrigatoriedade em seguir regras de isolamento social estritas, com concomitante fechamento de fronteiras impostas por governos de alguns países, até ao planejamento e à adoção de medidas de saúde para enfrentar a crise onde ainda está incipiente. O certo é que a Covid-19 impregnou-se no cotidiano de todos, de forma dominante e, talvez, sem precedentes.

Usando-se o buscador da internet Google com os termos "Covid-19", "Covid19", "Covid 19", "Covid-19 Brasil", "coronavirus" e "coronavirus Brasil" os números alcançam quase nove bilhões de resultados quando não se restringe ao Brasil, e cinco quando o país é especificado. São números que, provavelmente, impressionam qualquer céptico em relação à magnitude da enfermidade e alertam aqueles que estão cientes do impacto sobre

1 - Professora do Departamento de Cirurgia da Faculdade de Medicina da UFMG, médica da equipe ETERNA, Rede Mater Dei, Belo Horizonte, MG, Brasil. Presidente da Comissão Qualidade e Segurança em Cirurgia do Colégio Brasileiro de Cirurgiões, Editora-chefe da Revista do Colégio Brasileiro de Cirurgiões. 2 - Professor Adjunto de Técnica Operatória da Universidade Federal do Estado do Rio de Janeiro (UNIRIO), Rio de Janeiro, RJ, Brasil. Diretor de Publicações do Colégio Brasileiro de Cirurgiões. 3 - Professor do Departamento de Cirurgia da Pontifícia Universidade Católica do Paraná, Hospital Universitário Cajuru, Curitiba, PR, Brasil. Presidente do Colégio Brasileiro de Cirurgiões. 
a sociedade contemporânea global, principalmente aqueles que trabalham na área da saúde. Neste quesito, os médicos são não só os responsáveis por triar, atender e tratar aqueles acometidos pela doença mas também os questionados pela população sobre como comportar-se frente às mais diversas situações. Isso demanda conhecimento sobre a enfermidade e preparo para enfrentá-la, algo difícil em qualquer circunstância, pois trata-se de doença muito nova, que surgiu no final de 2019. A despeito desta realidade, em pouco mais de três meses, pode-se encontrar na literatura científica grande número de publicações que abordam a doença, nos diversos tipos de divulgação científica como experimentos in vitro, relatos de casos, uso de potenciais medicamentos e até revisão sistemática ${ }^{1}$.

No Pubmed há, conforme a palavrachave utilizada, entre 1300 a mais de 3000 artigos publicados, o que denota a relevância do assunto (figura 1)2. Por outro lado, a capacidade para ler, analisar e concluir sobre o que melhor se aplica à realidade de cada um é limitada, não só pelo pouco tempo, mas também por falta de conhecimento sobre o método científico correto. Há muito que Jonh loannidis vem questionando a deficiência metodológica da pesquisa e a falta de senso crítico dos profissionais da saúde ${ }^{3-6}$. De forma provocativa, em recente texto de opinião, loannidis volta sua "metralhadora metodológica" contra os dados apresentados até o momento sobre o Covid-197. Todas estas incertezas aumentam a responsabilidade daqueles que, talvez, melhor possam orientar tanto o público em geral como os médicos: os órgãos de saúde mundial e nacional, e as sociedades de especialidades. Saliente-se que esta tarefa é árdua e pode também ser marcada por controvérsias, uma vez que o que hoje parece ser lógico, amanhã poderá ser contestado. Afinal, as verdades transitórias sempre marcaram a Medicina.
PubMed Advanced Search Builder
PublMed.gov

User Guide

Add terms to the query box
ADD

Show Index

Search

Enter / edit your search query here

History and Search Details

$\downarrow$ Download 画 Delete

Search Actions Details Query

Results Time

\#3 $\quad \cdots \quad>\quad$ Search: "coronavirus surgery"

$414 \quad 05: 24: 31$

\#2 $\quad$-. $\quad>\quad$ Search: "new coronavirus surgery"

$59 \quad$ 05:24:06

$\# 1 \quad \cdots \quad>\quad$ Search: "covid-19 surgery"

$88 \quad 05: 23: 27$

Figura 1. Cópia da página do Pubmed.gov²7. 
O experiente e sábio Professor Saul Goldenberg há alguns anos indagou "Como editor de revista científica pergunto-me sempre: Como ficar convencido da veracidade de um artigo científico? Como discernir o verdadeiro do falso?"8. Ioannidis, por outro lado, afirma que a grande maioria dos trabalhos científicos é falsa ${ }^{6}$. Assim, muitos profissionais acreditam que a melhor forma para solucionar a avalanche de informação científica ${ }^{9}$ é ler revisões sistemáticas, metaanálises ou guias, principalmente de fontes conceituadas como a Cochrane, algo que também exige senso crítico. Afinal, as controvérsias sobre estas fontes, tidas como de qualidade, têm sido frequentemente questionáveis com embasamento científico 3,4,10-12. É certo que a possibilidade de produção de níveis de evidência elevados neste cenário é muito difícil. Entretanto, a própria Medicina Baseada em Evidências é definida como "o emprego consciencioso, explícito e judicioso da melhor evidência disponível na tomada de decisões sobre os cuidados de saúde de um paciente ${ }^{12 " . ~ E ~ e m b o r a ~}$ as publicações até o momento sejam fracas do ponto de vista de evidências, incluindo análise de ensaios clínicos ainda em andamento e tratamentos "off-label"; é o que temos à nossa disposição para enfrentarmos esta pandemia. Nos cabe somente, enquanto comunidade científica, senso crítico e temperança na análise destes dados.

De sorte que neste momento crítico instituições científicas devem assumir a liderança no esclarecimento de fatos e verdades, ainda que a evidência possa ser ténue e passível de mudanças. Neste sentido, o Colégio Brasileiro de Cirurgiões, com a maturidade de um nonagenário, por meio de seu veículo científico, a Revista do Colégio Brasileiro de Cirurgiões, propõe medidas de qualidade e segurança visando orientar os profissionais cirurgiões e os pacientes, tendo como base o que há disponível, até o momento, em cirurgia, na literatura científica ${ }^{13-17}$, nas diretrizes do Ministério da Saúde do Brasil $^{18}$, sob a liderança firme do cirurgião ortopedista Dr. Luiz Henrique Mandetta, e na página da Organização Mundial de Saúde ${ }^{19}$.

O fato é que os serviços de saúde devem estar preparados para situação emergencial de evolução rápida, que muda exponencialmente, a qualquer momento. Na verdade, os serviços de saúde deveriam ter, como princípio perene, estratégias e diretrizes de qualidade e segurança cirúrgica (20), abordando também temas relacionados aos desastres naturais, às guerras e pandemias, algo que na atual situação assume relevante pape ${ }^{13}$. De sorte que:

- Procedimentos cirúrgicos eletivos devem ser re-agendados para momento oportuno.

- Justificativa: 1) haver necessidade de espaços disponíveis para receber mais pacientes; 2) disponibilizar mais aparelhos de ventilação mecânica, em caso de demanda aumentada ${ }^{13}$; 3) prevenir eventos adversos em pacientes submetidos a procedimentos cirúrgicos de baixa morbimortalidade, mas que foram fatais em enfermos infectados pelo Covid-19, assintomáticos ${ }^{16}$.

- Traçar plano objetivo para realizar operações essenciais durante a pandemia, especificamente urgências e emergências.

- Justificativa: 1) atender a situações tais como trauma, hemorragias digestivas, infecções graves que demandam intervenção imediata ou quase imediata; 2) preparar e orientar toda a equipe assistencial em como proceder, protegendo-se eficazmente por meio de uso de equipamentos de proteção individual (EPI); 3) permitir o mínimo de pessoas necessárias em sala cirúrgica, evitando assim o risco de maior de contaminação de outros profissionais. Na verdade, este quesito 
deveria ser parte do treinamento em qualidade e segurança para atender situações de desastres e pandemias ${ }^{13}$.

- Definir, se possível, considerando o tipo de hospital e a dinâmica assistencial, salas específicas para a realização de operações, com orientações sobre quesitos importantes a serem seguidos nesta fase, colocados de forma visível para todos.

- Justificativa: 1) diminuir o tráfego de pessoas e objetos; 2) garantir ao paciente menos contato com potenciais transmissores da doença ${ }^{21}$

- Ter cuidado rigoroso, durante as de operações, laparoscópicas ou abertas, com o uso aparelhos de aspiração, cauterização, insuflação. A pressão do pneumoperitónio deve ser mantida o mais baixa possível, respeitando a segurança da exposição anatómica da região a ser operada. O cautério deve ser usado com a menor potência capaz de realizar adequada cauterização.

- Justificativa: minimizar a dispersão de partículas contaminantes ${ }^{17}$;

- Realizar desinfecção rigorosa da sala e dos materiais utilizados conforme as orientações dos órgãos sanitários locais ${ }^{18}$.

- Justificativa: 1) garantir a segurança do próximo paciente e da equipe assistencial.

- Limitar o número de visitas aos pacientes operados, principalmente em unidades de terapia intensiva (se necessário, até avaliar a suspensão).

- Justificativa: 1) diminuir o tráfego de pessoas e objetos; 2) garantir ao paciente menor contato com potenciais transmissores da doença ${ }^{15}$.

- Garantir, nos casos de trauma, que a equipe realize o processo assistencial desde a triagem, o primeiro atendimento no local do trauma, o transporte e a admissão hospitalar em conformidade com o descrito anteriormente, principalmente, no tocante ao uso de EPIs ${ }^{14}$.

- Justificativa: 1) diminuir a disseminação do vírus entre os profissionais de saúde maiormente expostos a secreções, considerando todos os pacientes como potenciais transmissores ; 2) minimizar o risco de transmissão para o paciente, a partir de profissionais de saúde assintomáticos; 3) reorganizar os horários e plantões das equipes antevendo o risco de cansaço excessivo e adoecimento concomitante de vários membros da equipe médica, o que poderia afetar o desempenho, e a oferta de número adequado de profissionais.

- Desenvolver estratégias de apoio aos profissionais de saúde, principalmente, aqueles com filhos em idade escolar, para que as melhores condições psicológicas possíveis de trabalho sejam alcançadas.

- Justificativa: 1)as aulas estão suspensas; 2) potenciais cuidadores, como avós, são grupo de risco para maior morbidade relacionada ao Covid-19, logo não devem ficar responsáveis pelos cuidados das crianças $^{19}$

- Apoiar, neste momento crítico, a orientação do Conselho Federal de Medicina ofício $n^{\text {o. }} 1756 / 2020^{22}$ sobre teleorientação, telemonitoramento e a teleinterconsulta.

- Justificativa: 1) necessidade de apoio remoto a pacientes em isolamento; 2) apoiar sob orientação e supervisão médica o monitoramento ou vigência à distância de parâmetros de saúde ou doença; 3) possibilitar a troca de informações e opiniões entre médicos, para auxílio diagnóstico e terapêutico 
- Re-marcar operações somente quando sugerido pelos órgãos de saúde nacionais ${ }^{18}$.

O Colégio Brasileiro de Cirurgiões ciente de que as informações são muitas, mas a evidência científica ainda é fraca sugere, essencialmente, medidas de qualidade e segurança gerais para com os pacientes e equipes de saúde. Os profissionais de saúde não devem ser vistos como heróis ${ }^{23}$, mas sim como seres humanos que escolheram como profissão o cuidado ao outro, sendo ensinados para tal, mas que infelizmente, não são rotineiramente treinados para enfrentar situações de crise e muito menos, têm preparo científico suficiente para questionar a avalanche de trabalhos que são publicados ${ }^{12,24-26}$.

\section{A B S T R A C T}

The current world Covid-19 pandemic has been the most discussed topic in the media and scientific journals. Fear, uncertainty, and lack of knowledge about the disease may be the significant factors that justify such reality. It has been known that the disease presents with a rapidly spreading, it is significantly more severe among the elderly, and it has a substantial global socioeconomic impact. Besides the challenges associated with the unknown, there are other factors, such as the deluge of information. In this regard, the high number of scientific publications, encompassing in vitro, case studies, observational and randomized clinical studies, and even systematic reviews add up to the uncertainty. Such a situation is even worse when considering that most healthcare professionals lack adequate knowledge to critically appraise the scientific method, something that has been previously addressed by some authors. Therefore, it is of utmost importance that expert societies supported by data provided by the World Health Organization and the National Health Department take the lead in spreading trustworthy and reliable information. The Brazilian College of Surgeons suggests in this document various initiatives that may help surgeons, healthcare providers, and patients who will have to face a surgical event under the pandemic.

Keywords: Pandemics. General Surgery. Preoperative Care. Intraoperative Care. Postoperative Care. Disaster Planning.

\section{REFERÊNCIAS}

1. Salehi S, Abedi A, Balakrishnan S, Gholamrezanezhad A. Coronavirus Disease 2019 (COVID-19): A Systematic Review of Imaging Findings in 919 Patients. AJR Am J Roentgenol. 2020:1-7.

2. Pubmed [Available from: https://pubmed.ncbi.nlm. nih.gov/.

3. Ioannidis JPA. Randomized controlled trials: Often flawed, mostly useless, clearly indispensable: A commentary on Deaton and Cartwright. Soc Sci Med. 2018.

4. Ioannidis JPA. Hijacked evidence-based medicine: stay the course and throw the pirates overboard. J Clin Epidemiol. 2017;84:11-3.

5. Ioannidis JP. Evidence-based medicine has been hijacked: a report to David Sackett. J Clin Epidemiol. 2016;73:82-6.

6. Ioannidis JP. Why most published research findings are false. PLoS Med. 2005;2(8):e124.
7. loannidis JPA. A fiasco in the making? As the coronavirus pandemic takes hold, we are making decisions without reliable data Statnew: Boston Globe; 2020 [Available from: https:// www.statnews.com/2020/03/17/a-fiasco-inthe-making-as-the-coronavirus-pandemic-takeshold-we-are-making-decisions-without-reliabledata/?fbclid=IwAROr5W-fu7C4Qi19bUdiW_ ekgbTu6HpP98xx5BBaZjL1bZn5QjlvaVbm3yc.

8. Goldenberg S. [Reflections on the truth]. Acta Cir Bras. 2007;22(6):420-1.

9. C A. The End of Theory: The Data Deluge Makes the Scientific Method Obsolete https://www.wired. com/2008/06/pb-theory/2008 [

10. Askheim C, Sandset T, Engebretsen E. Who cares? The lost legacy of Archie Cochrane. Med Humanit. 2017;43(1):41-6.

11. Noone KJ. Beware the impact factor. Ambio. 2016;45(5):513-5. 
12. Correia M. Are we capable of separating the wheat from the chaff when assessing meta-analyses? Clin Nutr. 2020;39(3):705-7.

13. Brindle M, Gawande A. Managing COVID-19 in Surgical Systems. Ann Surg. 2020; accepted(on line).

14. Surgeons TcotQpotACo. Maintaining trauma Center Acess and Care during the Covid-19 Pandemic: Guidance Document for Trauma Medical Directors 2020 [Available from: https://www.facs.org/qualityprograms/trauma/maintaining-access.

15. Surgeons ACo. Covid-19 Recommendations for Management of Elective Surgical Procedures [Available from: https://www.facs.org/about-acs/ covid-19/information-for-surgeons.

16. Aminian A, Safari S, Razeghian_Jahromi A, Ghorbani M, Delaney CP. Covid-19 Outbreak and Surgical Practice: Unexpected Fatality in Perioperative Period. Ann Surg. 2020; on line.

17. Zheng $M H$, boni $L$, Fingerhut $A$. Mlnimally invasive surgery and the novel coronavirus outbreak: lessons learned in China and Italy. Ann Surg. 2020; on line.

18. Saúde Md. 2020 [cited 2020 March, 22nd 2020]. Available from: https://saude.gov.br/.

19. Organization $W H$. Rolling updates on coronavirus disease (COVID-19). 2020.

20. Saúde Md. Cirurgias seguras salvam vidas Rio de Janeiro: Organização Pan-Americana da Saúde ; Ministério da Saúde ; Agência Nacional de Vigilância Sanitária; 2009 [Available from: http://bvsms. saude.gov.br/bvs/publicacoes/seguranca_paciente_ cirurgias_seguras_guia.pdf.
21. Zheng $M H$, Boni $L$, Fingerhut $F$. Rolling updates on coronavirus disease (COVID-19) 2020 [Available from: https://www.who.int/emergencies/diseases/ novel-coronavirus-2019/events-as-they-happen.

22. OFíCIO CFM No 1756/2020 - COJUR - Telemedicina, (2020).

23. Herós em guerra. Veja. 2020 March, 25th, 2020.

24. Grech $\vee$, Rizk DEE. Increasing importance of research metrics: Journal Impact Factor and h-index. Int Urogynecol J. 2018;29(5):619-20.

25. Karabulut N. Inaccurate Citations in Biomedical Journalism: Effect on the Impact Factor of the American Journal of Roentgenology. AJR Am J Roentgenol. 2017;208(3):472-4.

26. Ioannidis JP. The Mass Production of Redundant, Misleading, and Conflicted Systematic Reviews and Meta-analyses. Milbank Q. 2016;94(3):485-514.

27. "Coronavirus surgery" [Pubmed]. 2020 [Available from: https://pubmed.ncbi.nlm.nih. gov/?term=\%22coronavirus+surgery $\% 22$.

Recebido em: 23/03/2020

Aceito para publicação em: 23/03/2020

Conflito de interesse: nenhum.

Fonte de financiamento: nenhuma.

\section{Endereço para correspondência:}

M. Isabel T. D. Correia

E-mail: isabel_correia@uol.com.br

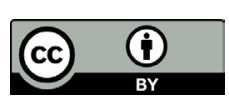

